\title{
Kalman Filter Applied to a Active Queue Management Problem
}

\author{
Jyoti Pandey ${ }^{1}$ and Prof. Aashih Hiradhar ${ }^{2}$ \\ Department Of Electronics And Telecommunication, ${ }^{12}$, C.V.Raman University, Bilaspur (C.G.),
}

\begin{abstract}
The Kalman Filter (KF) is used to optimally estimate system states from the sequential noisy measurements of the outputs. On the other hand, real-time systems are often modeled with uncertainties and time-delays. Developing KF algorithms for such systems is an important problem to obtain optimal state estimates by utilizing the information on uncertainties and time-delays. First, designing of KF for nominal discrete-time systems is studied. Considering the covariance of the error in the estimation the KF algorithm is derived which is further tested on a numerical example. A designed $K F$ is tested on a real-time problem i.e. Active Queue Management (AQM) problem.In Internet routers, Active Queue Management (AQM) is a technique that consists in dropping or Explicit Congestion Notification (ECN) marking packets before a router's queue is full.
\end{abstract}

Keywords: Active Queue Management,Explicit Congestion Notification, discrete time system, time-delay.

\section{Introduction}

Basically the KF is an estimator which estimate the future state from the series of noisy measurement for the given system state. The KF provides a method for constructing an optimal estimate of the system state. $\mathrm{KF}$ is an efficient recursive filter that estimates the state of a linear dynamic system from series of noisy measurement.

The KF is a recursive estimator means that only the estimated state from the previous step and the current measurement are needed to compute the estimate for the current state. The KF operates by propagating the mean and covariance of the state through time. Basically the KF has two distinct phase time update \& measurement update. The time update uses the state estimates from the previous time step to produce an estimate of the state at the current time step. The measurement update information at the current time step is used to refine this prediction at new.The Kalman filter is a tool that can estimate the variables of a wide range of processes. In mathematical terms we would say that a Kalman filter estimates the states of a linear system. The Kalman filter not only works well in practice, but it is theoretically attractive because it can be shown that of all possible filters, it is the one that minimizes the variance of the estimation error. Kalman filters are often implemented in embedded control systems because in order to control a process, you first need an accurate estimate of the process variables.

The Kalman filter is a mathematical power tool that is playing an increasingly important role in computer graphics as we include sensing of the real world in our systems. The good news is you don't have to be a mathematical genius to understand and effectively use Kalman flters. This tutorial is designed to provide developers of graphical systems with a basic understanding of this important mathematical tool.

The process of finding the "best estimate" from noisy data amounts to "filtering out" the noise.

However a Kalman filter also doesn't just clean up the data measurements, but also projects these measurements onto the state estimate.

\section{Kalman Filtering For Linear Discrete Time System}

Kalman Filter (KF) is a numerical method used to track a time-varying signal in the presence of noise. It is the problem of estimating the instantaneous state of a linear system from a measurement of outputs that are linear combinations of the states but corrupted with Gaussian white noise. The resulting estimator is statically optimal with respect to a quadratic function of estimation error. From the mathematical point view, the KF is a set of equations that provides an efficientrecursive computational solution of the linear estimation problem. The filter is very powerful in several aspects. It can be suitably used to either of smoothening, estimating or predicting respectively the past, the present and the future states. This may be achieved even when precise characteristics of the modeled system are unknown, i.e., the case of uncertain systems. The KF is an extremely effective and versatile procedure for combining noisy sensor outputs to estimate the state of the system with uncertain dynamics. When applied to a physical system, the observer or filter will be under the influence of two noise sources: (i) Process noise, (ii) Measurement noise.The estimate of the state is specified by its conditional probability density function. The purpose of a filter is to compute the state estimate, while an optimal filter minimizes the spread of the estimation error probability density. A recursive optimal filter propagates the conditional probability density function from one sampling instant to the next, keeping in view the system 
dynamics and inputs, and it incorporates measurements and measurement error statistics in the estimate. Therefore, the recursive generation of the mean and covariance in finite time can be expressed as the following five steps: (i) State estimate extrapolation (Propagation), (ii) Covariance estimation extrapolation (Propagation), (iii) Filter gain computation, (iv) State estimate update, (v) Covariance estimate update.

We use a KF to estimate the state $x_{k} \in \mathfrak{R}^{n}$ of a discrete time controlled system. The system is described by a linear stochastic difference equation.

$$
\begin{aligned}
& x_{k+1}=A x_{k}+B w_{k} \\
& y_{k}=C x_{k}+v_{k}
\end{aligned}
$$

where, $x_{k} \in \mathfrak{R}^{n}$ is the system state, $y_{k} \in \mathfrak{R}^{m}$ is the measured output, $w_{k} \in \mathfrak{R}^{q}$ is the process noise, $v_{k} \in \mathfrak{R}^{p}$ is the measurement noise. In the following $v_{k}$ and ${ }^{w_{k}}$ will be regarded as zero

mean, uncorrelated white noise sequence with covariance $R_{k}$ and $Q_{k}$.

$v_{k}=N\left(0, R_{k}\right)$

$w_{k}=N\left(0, Q_{k}\right)$.

The matrix $A \in \mathfrak{R}^{n \times n}$ in the difference Equation (2.1) is the dynamics matrix which relates the sate at time step $k_{\text {to the sate at time step }} k+1$. The matrix $B \in \mathfrak{R}^{n \times 1}$ called noise matrix. The matrix $C \in \mathfrak{R}^{m \times m}$ in the measurement Equation (2.2) relates the state measurement $y_{k}$.When the measurement error covariance $R_{k}$ approaches zero, the weighting by $K_{f}$ lets the actual measurement ${ }^{y_{k}}$ be "trusted" more and more, while the predicted measurement $C_{k} \hat{x}_{k}^{-}$is trusted less and less. On the other hand, the actual measurement ${ }^{k_{k}}$ is trusted less and less as the a priori estimate error covariance $P_{k}^{-}$approaches zero, and the predicted measurement $C_{k} \hat{x}_{k}^{-}$is trusted more and more.

The KF algorithm can be seen as a form of feedback estimation. The set of the KF equation can be separated in two groups:

1. Time update equations

2. Measurement update equation

The time update equations project the current state and the error covariance estimates forward in the time to obtain a priori estimates for the next time step. The measurement update equations handle the feedback. In other words, it incorporates a new measurement into the a priori estimate to obtain a corrected a posterior estimate. Therefore the time update equations are predicator equations, and the measurement update equations are corrector equations. That is, the KF is a predictor-corrector algorithm to provide a recursive solution to the discrete time linear system, as shown in Fig. 2.1.

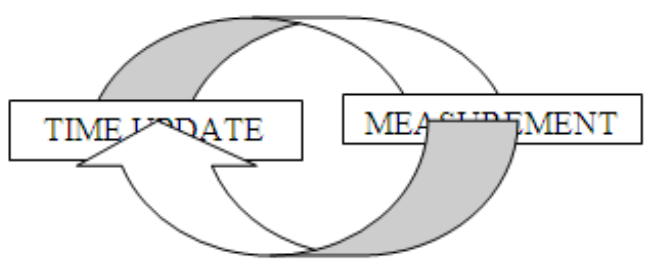

Figure 2.1: Discrete Klaman Filter Cycle

The time and measurement update equations are presented below:

The time updates equations:

$\hat{x}_{k+1}^{-}=A \hat{x}_{k}+B u_{k}$ (2.5)

$P_{k+1}^{-}=A P_{k} A_{k}^{T}+Q_{k}$

The measurements update equations: 


$$
\begin{aligned}
& K_{f}=P_{k}^{-} C_{k}^{T}\left(C_{k} P_{k}^{-} C_{k}^{Y}+R_{k}\right)^{-1} \\
& \hat{x}_{k}=\hat{x}_{k}+K_{f}\left(y_{k}-C_{k} \hat{x}_{k}^{-}\right)(2.8) \\
& P_{k}=\left(I-K_{f} C_{k}\right) P_{k}^{-}
\end{aligned}
$$

The time-update equation projects the state estimate and covariance from time step $k$ to step $k+1$.

To compute the Kalman Gain (KG) $K_{f}$ is the first job in the measurement update equations. Then $y_{k}$ is obtained by actual measurement of the system. Incorporating the actual measurement and the estimated one in equation(2.7), we generate a posterior estimate. The last step is to compute a posterior error covariance. This is the recursive operation of the KF. A complete picture of the operation of the KF is illustrated in Fig. 2.2, after each time and measurement update pair, the recursive algorithm is repeated with the previous a posterior estimates to predict the new a priori estimates. This recursive nature is the biggest advantage of the KF. This makes the practical implementation of the KF much easier and feasible then the implementation of the Wiener filter, because the Weiner filter obtains its estimates by using all of the precedent data directly. In contrast, the $\mathrm{KF}$ only uses the immediately previous data to predict the current states [10]. The standard KF algorithm is shown in Fig. 2.2.

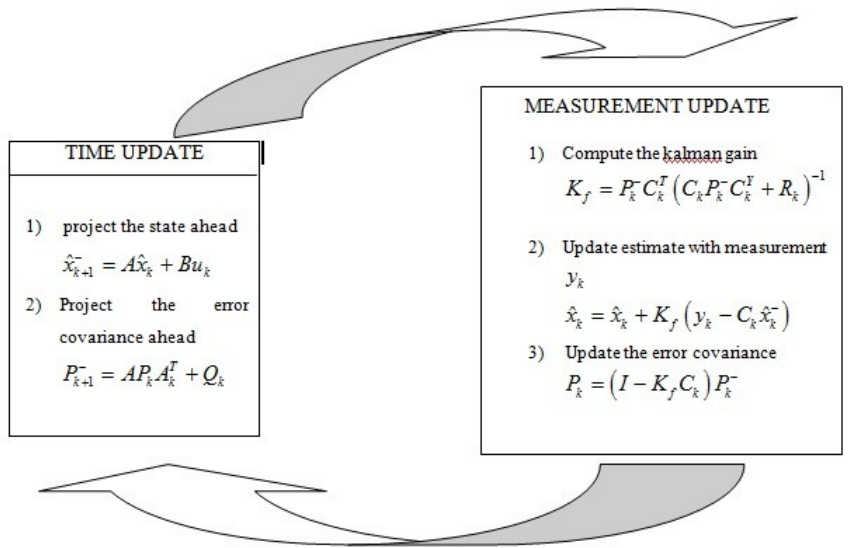

Figure 2.2: Kalman Filter Algorithm

III. Active Queue Management Problem

In Internet routers, Active Queue Management (AQM) is a technique that consists in dropping or Explicit Congestion Notification (ECN) marking packets before a router's queue is full [8].

An Internet router typically maintains a set of queues, one per interface, that hold packets scheduled to go out on that interface. Historically, such queues use a drop-tail discipline: a packet is put onto the queue if the queue is shorter than its maximum size (measured in packets are in bytes), and dropped otherwise. Active queue disciplines drop or mark packets before the queue is full. Typically, they operate by maintaining one or more drop/mark probabilities, and probabilistically dropping or marking packets even when the queue is short. The uniqueness of our approach comes from the use of a recently developed dynamic model of the transmission control protocol (TCP) which enables application of control principles to address the basic feedback nature of AQM [6].

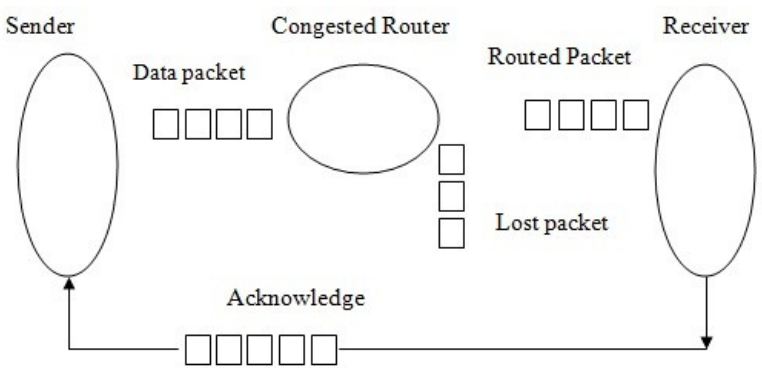

Figure 3.1: A Sender Receiver Connection 


\section{Results \& Simulations}

4.1 Simulation Result of Kalman Filter

Consider the following discrete time system,

$$
\begin{aligned}
& x_{k+1}=\left[\begin{array}{cc}
0 & -0.5 \\
1 & 1
\end{array}\right] x_{k}+\left[\begin{array}{c}
-6 \\
1
\end{array}\right] w_{k} \\
& y_{k}=\left[\begin{array}{ll}
-100 & 10
\end{array}\right] x_{k}+v_{k}
\end{aligned}
$$

Note that the above system is of the form of system with

$$
P=S=\left[\begin{array}{ll}
1 & 0 \\
0 & 1
\end{array}\right], W=1, V=1
$$

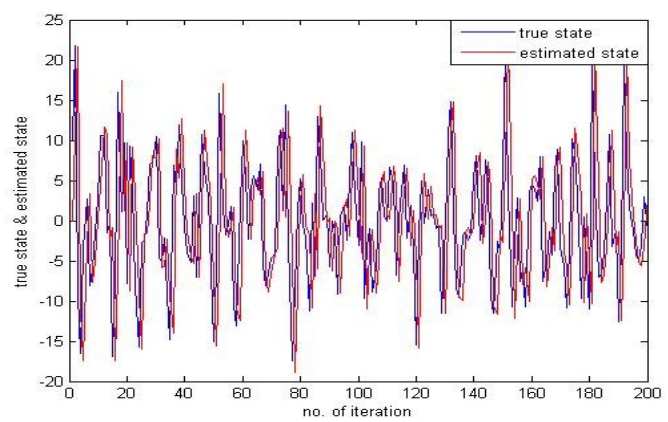

Figure 4.1: No. of Iteration vs. True State and Estimated State

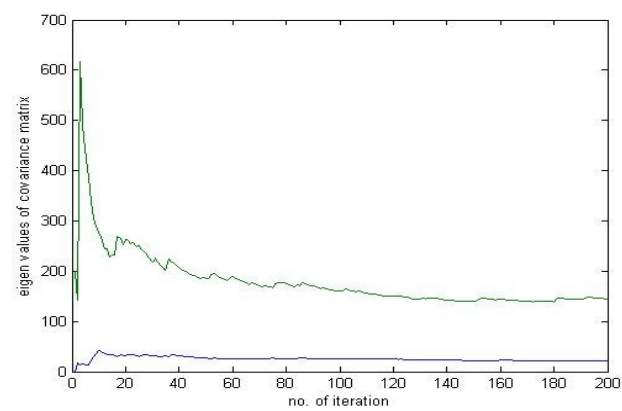

Figure 4.2: No. of Iteration vs. Eigen Value of Covariance Matrix

Fig.4.1 shows the estimated states by KF and true state of a system which is considered. And we observe from the figure that estimated state is nearly equal to true state with some error.

Fig.4.2 shows when the number of iteration increases the estimation error decreases and becomes steady after a finite interval of time. The value of covariance of estimation error is shown below

$$
\operatorname{Cov}\left(e_{k}\right)=\left[\begin{array}{cc}
76.2930 & -53.0900 \\
-53.0900 & 75.6653
\end{array}\right]
$$

4.2 State Estimation using Kalman Filter in active queue management problem

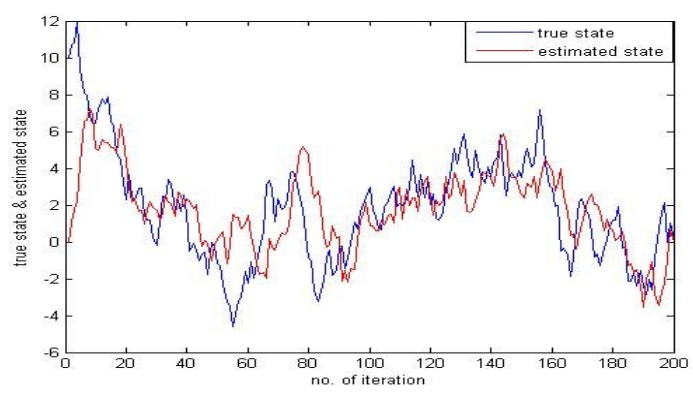

Figure 4.3: Number of iteration and true state $\&$ estimated state 


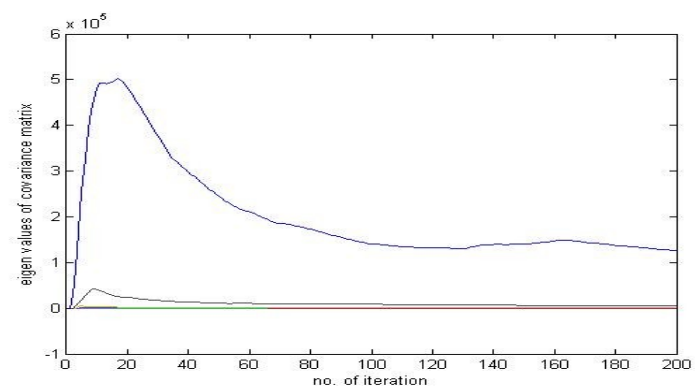

Figure 4.4: Number of iteration Eigen values of covariance matrix

\section{Applications Of Kalman Filter}

Kalman filter is used in Inertial navigation and guidance, Global positioning system, Target tracking, Communication, signal processing,. Fault diagnosis, Channelequalization, Economics, Navigation, Many computer vision application and Feature tracking.

\section{Limitations Of Kalman Filter}

One of the problem with the Kalman filter is that they may not robust against modeling uncertainties. The Kalman filter algorithm is the optimal filter for a system without uncertainties. The performance of a KF may be significantly degraded if the actual system model does not match the model on which the KF was based.

\section{Conclusion}

$\mathrm{KF}$ is a powerful tool to estimate states of a system under noisy output measurements. In this chapter, a formulation has been presented for the design of KF for linear systems without considering the time-delay and some basic ideas on KF. For estimation, prediction \& reduction of error the KF performance is satisfactory. The $\mathrm{KF}$ is the most widely used filter for its better performance. It is a very simple and intuitive concept with good computational efficiency. But when uncertainty and time delay is included then the performance of KF may degraded, so RKF is considered which is robust against large parameter uncertainty and time delay. In coming chapter a performance comparison between RKF and KF is obtained for linear discrete-time uncertain system.secondary, a real time problem i.e. AQM is considered. Initially the system was in continuous time domain then it is converted to discrete time domain. And the discritized form is transformed to another model for easy implementation of KF. From the Fig. 4.3 and 4.4 we see that the estimated state is approximate to the true state and with the increases of number of iteration the error will be reduce.

\section{References}

[1]. Antonis Papachristodouluo and Ali Jadbaaie, "Delay Robustness of Nonlinear Internet Congection Control Schemes", IEEE Transaction on aytomatic control, Volume 55, pp- 1421-1427,(2010).

[2]. Mital A Gandhi, and Lamine Mili, "Roust Kalman Filter based on a Generalized Maximum- Likegood-Type Estimator", IEEE Transaction on Signal Processing, Volume 58, pp-2509-2520,(2010).

[3]. Rodrigo Fontes Souto and Joao Yoshiyuki Ishihara, "Robust Kalman Filter for Discrete-Time Systems With Correlated Noise", Ajaccio, France, 2008.

[4]. Ashvin Lakshmikantha, Carolyn L. Beck, and R. Srikant, "Robustness of Real and Virtual Queue- Based Active Queue Management Schemes", IEEE/ACM Transaction on networking, Volume 13, pp- 81-93,(2005).

[5]. X.Lu, H.Zhang and W.Wang, "Kalman Filtering for Multiple Time Delay System", Automatica, Volume 41, pp- 1455-1461 (2005).

[6]. Tansu Alpcan, and Tamer Basar, "A Gloally Stable Adaptive Congestion Control Scheme for Internet-Style Networks With Delay ", IEEE/ACM Transaction on networking, Volume 13, pp-1261-1274,(2005).

[7]. Z.Wang, J.Lam and X.Liu, "Robust Kalman filtering for Discrete-Timr Markovian Jump Delay Sustem", IEEE Signal Processing Letters, Volume 11, pp- 659-662 (2004).

[8]. C.V.Hollot, V.Mishra, D.Towsley, and W.Gong "Analysis and Design of Controllers for AQM Routers Supporting TCP Flows", IEEE Transactions on Automatic Control, Volume 47, pp- 954-959, (2002).

[9]. V.Mishra, W.Gong, and D.Towsley, "Fluid based analysis of a network of AQM routers supporting TCP flows with an application to RED", Proceeding ACM/SIGCOMM, (2000).

[10]. V.Mishra, W.Gong, and D.Towsley, "Fluid based analysis of a network of AQM routers supporting TCP flows with an application to RED", Proceeding ACM/SIGCOMM, (2000). 